\title{
Hypoxic-ischaemic injury - the 'white cerebellum sign' versus the true 'reversal sign'
}

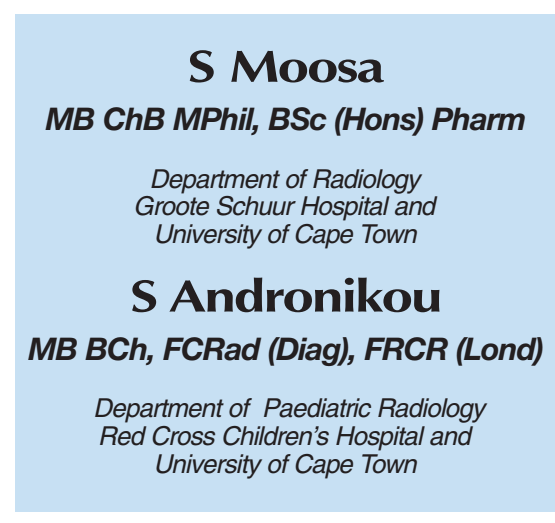

\section{Introduction}

A small percentage of patients who suffer a global cerebral hypoxic/ ischaemic injury develop reversal of the normal density relationship of grey and white matter on CT scans. ${ }^{1,2}$ The neuroradiology texts are confusing in their description of the CT appearance of the 'reversal sign' ${ }^{3,4}$ which is a feature related to severe hypoxia (birth asphyxia, cardiopulmonary arrest), trauma (child abuse) and infection. ${ }^{1,2,5}$ We present two paediatric cases with this type of injury, one of which demonstrates the 'white cerebellum sign' and the other the true 'reversal sign', in order to demonstrate the imaging differences.

\section{Case reports}

\section{Case 1}

A CT scan was performed on a 4- year-old child who presented with acute convulsions and herpetic ulcers with an associated metabolic acidosis. The scan showed global hypodensity of the supratentorial parenchyma with effacement of the grey-white interface and of the central and peripheral cerebrospinal fluid (CSF) spaces (Fig. 1). The density of the cerebellum and other infratentorial structures is preserved and shows a relatively hyperdense appearance that contrasts with the supratentorial structures. This is an example of the 'white cerebellum sign'.

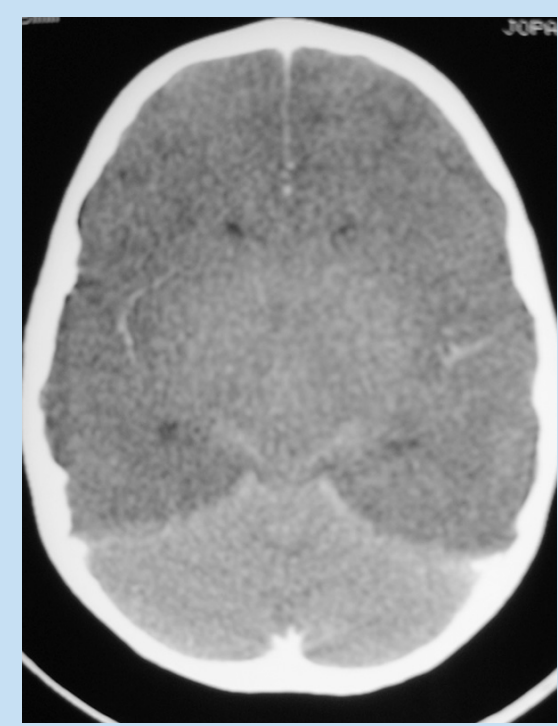

Fig. 1. White cerebellum sign. There is global hypodensity of the supratentorial brain with relative hyperdensity of the infratentorial compartment. There is also obliteration of the surface and central CSF spaces in keeping with the severe oedema.

\section{Case 2}

In contrast, the CT scan in the second case, a young patient with streptococcal meningitis, shows hypodensity of the peripheral cortex with sparing of the central white matter, grey matter nuclei and posterior fossa structures, and reversal of the greywhite difference (GWD) (Figs 2a, b). This is consistent with cortical laminar necrosis, which later resulted in the linear calcification of the cortex seen on a long-term follow-up CT scan (Fig. 3).

\section{Discussion}

The pathogenesis of the reversal sign is not well understood. ${ }^{2}$ Several factors may play a role.

\section{Mechanical factors}

1. Preservation of central areas of the brain has been demonstrated in animal experiments. Transtentorial herniation during the acute stage of oedema relieves the pressure on the expanding cerebral mass as brain is herniated through the incisura. Perfusion improves centrally and prevents necrosis. ${ }^{2,5}$

\section{Metabolic factors}

1. Anoxia and ischaemia elevate brain glucose. This hyperglycaemic state preferentially damages the cortex and basal ganglia. ${ }^{2,5}$

2. Hypoxia limits ATP production and disrupts the sodium pump. The influx of water and sodium into cells causes cytotoxic oedema. Later, the water content rises in the extracellular and intracellular spaces (vasogenic oedema). Low density changes appear in the areas most vulnerable to hypoperfusion. 


\section{CASE REPORT}
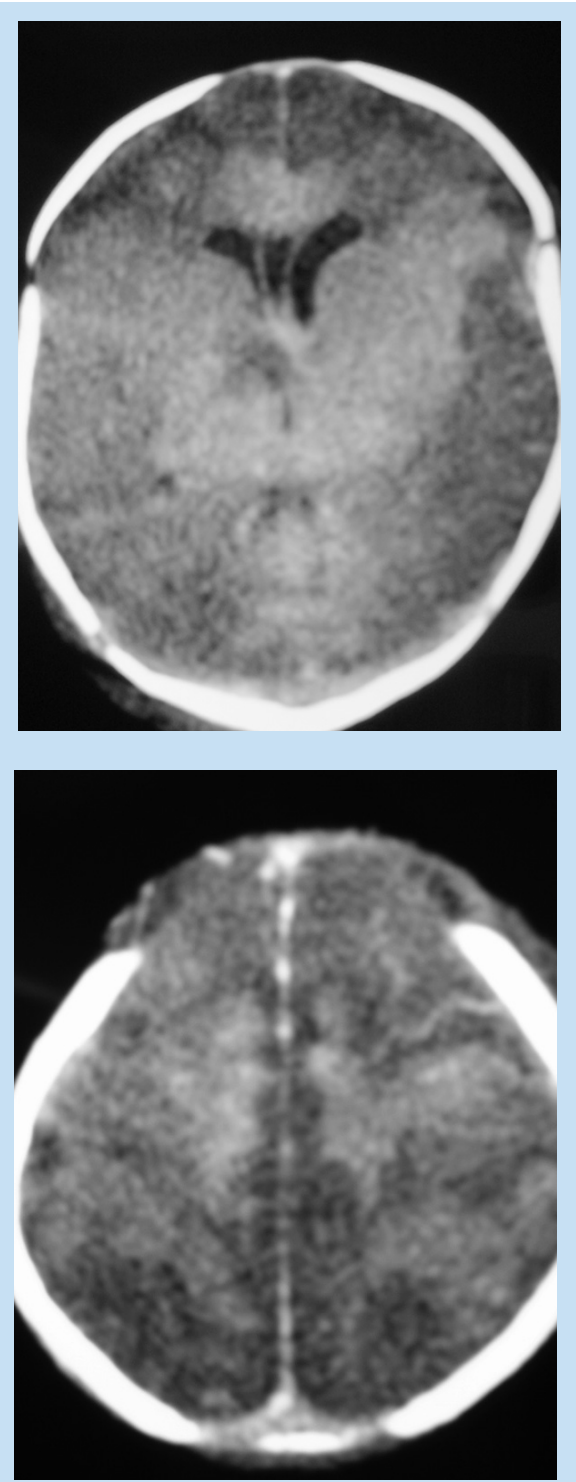

Fig. $2 a$ and $b$. Reversal sign. There is central hyperdensity compared with the peripheral cortex, involving the thalami and central white matter, as well as in the infratentorial structures.

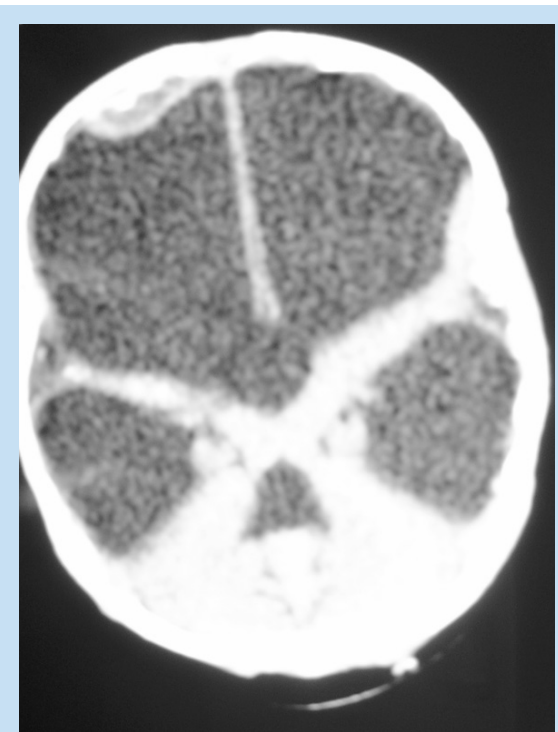

Fig. 3. The long-term follow-up CT in patient 2 demonstrates severe cortical and white matter losses, as well as peripheral cortical linear calcification in keeping with cortical laminar necrosis.

\section{Metabolic demand}

1. The basal ganglia and cortex have high metabolic activity, and are vulnerable to tissue hypoxia and hypoperfusion. Cortex and basal ganglia density decrease with loss of normal GWD. Sometime the grey density loss is so great that normal contrast is reversed, i.e. the reversal sign. ${ }^{5}$

\section{Haemodynamic changes}

1. Increased density of white-matter is caused by distended medullary veins and central venous congestion, contrasting with the peripheral cortical oedema. ${ }^{1,2,5}$ During hypoxia, hypoperfusion and oedema, thrombi are formed in these veins. When perfusion is established again, the outflow is obstructed and these veins become distended. ${ }^{5}$

The reversal sign is associated with a poor prognosis. ${ }^{1,2,5}$ One-third of patients demonstrating this sign on CT scans will die. The remainder suffer severe permanent brain damage, later developing diffuse atrophy and cystic encephalomalacia. ${ }^{2,5}$ It is therefore very important to recognise the features described above for diagnostic, therapeutic and prognostic purposes.

\section{References}

1. Bird CR, Drayer BP, Gilles FH. Pathophysiology of 'reverse' edema in global cerebral ischemia. Am J Neuroradiol 1989; 10: 95-98.

2. Han BK, Towbin RB, De Courten-Meyers G, McLaurin RL, Ball WS jun. The reversal sign: Effect of anoxic/ischemic cerebral injury in children. Am J Neuroradiol 1989; 10: 1191-1198.

3. Barkovich AJ. Brain and spine injuries. In: Barkovich AJ, ed. Pediatric Neuroradiology. 3rd ed. Pennsylvania: Lippincott Williams and Wilkins, 2000.

4. Osborne GO. Craniocerebral trauma. In: Osborne GO, ed. Diagnostic Neuroradiology. St Louis, Missouri: Mosby, 1994.

5. Vergote G, Vandeperre H, De Man R. The reversal sign. Neuroradiology 1992; 34: 215-216. 